\title{
Correlated Wishart Matrices and Critical Horizons
}

\author{
Zdzisław Burda, Andrzej Görlich, Jerzy Jurkiewicz, and Bartłomiej Wacław \\ Mark Kac Center for Complex Systems Research and Marian Smoluchowski Institute of Physics, \\ Jagellonian University, Reymonta 4, 30-059 Kraków, Poland
}

\begin{abstract}
We discuss a practical method to determine the eigenvalue spectrum of the empirical correlation matrix. The method is based on the analysis of the behavior of a conformal map at a critical horizon which is defined as a border line of the physical Riemann sheet of this map. The map is a convenient representation of the Marčenko-Pastur equation.
\end{abstract}

The relation between the eigenvalue spectrum of the empirical covariance matrix and the spectrum of the underlying covariance matrix plays an important role in many research areas ranging from physics, telecommunication 1], to information theory 2] and quantitative finance [3], and has attracted a great attention recently.

The problem can be mathematically formulated as follows. Suppose we have a statistical system with $N$ degrees of freedom $\mathbf{x}=\left(x_{i}\right), i=1, \ldots, N$. We collect $T$ independent samples of $\mathbf{x}$, each being a certain realization $\mathbf{X}_{\alpha}=\left(X_{i}\right)_{\alpha}$ of $\mathbf{x}, \alpha=1, \ldots, T$. The data are stored in a rectangular matrix $\mathbf{X}=\left(X_{i \alpha}\right)$ of dimension $N \times T$. The covariance matrix 12] C: $C_{i j}=\left\langle x_{i} x_{j}\right\rangle$ can be estimated from the data as $\mathbf{c}=(1 / T) \mathbf{X X}^{\tau}: c_{i j}=(1 / T) \sum_{t} X_{i t} X_{j t}$, where $\mathbf{c}$ is empirical correlation matrix. Here $\mathbf{X}^{\tau}$ stands for the transpose of $\mathbf{X}$. For $r \equiv N / T \rightarrow 0(N=$ const, $T \rightarrow \infty)$ the empirical covariance matrix $\mathbf{c}$ perfectly approximates C. However, in practice, the number of samples $T$ is finite. One is therefore interested in the relation between $\mathbf{c}$ and $\mathbf{C}$, in particular how well the eigenvalue distribution of $\mathbf{c}$ approximates the eigenvalue distribution of $\mathbf{C}$ for finite $r$.

One can formulate the problem in terms of random matrix theory [4, 5]. One can think of $\mathbf{c}=(1 / T) \mathbf{X X}^{\tau}$ as a matrix constructed of real [13] gaussian random matrix $\mathbf{X}$. The only requirement which one has to impose on the probability measure for $\mathbf{X}$ is that the two-point correlations are:

$$
\left\langle X_{i \alpha} X_{j \beta}\right\rangle=\delta_{\alpha \beta} C_{i j}
$$

Such an ensemble of matrices $\mathbf{X}$ is called correlated Wishart ensemble [4, 5, 6]. The delta $\delta_{\alpha \beta}$ in the last equation tells us that the samples are uncorrelated, while $C_{i j}$ that the degrees of freedom are correlated according to the covariance matrix $\mathbf{C}$.

The relation between the spectral density of $\mathbf{c}$ and $\mathbf{C}$ is given by the Marčenko-Pastur equation [7]. This equation has been intensively studied in the mathematical literature [8, 9]. The corresponding equations for correlated samples, that is for the case when the delta $\delta_{\alpha \beta}$ is replaced by a symmetric matrix $A_{\alpha \beta}$ in (1), have been derived recently [10] using a diagrammatic technique [4, 11].

The purpose of the present paper is twofold. Firstly, we want to describe a practical method to calculate the eigenvalue spectrum of the estimator c. Some similar methods have been discussed in the literature [8, 9]. However, we believe that a conformal map representation [5] used here leads to a particularly simple and effec- tive practical method. Secondly, the eigenvalue smearing and noise dressing observed in the empirical correlation matrix $\mathbf{c}$ have a simple interpretation in terms of the conformal map, which we want to present.

In order to determine the relation between the eigenvalue densities of the covariance matrices $\mathbf{c}$ and $\mathbf{C}: \rho_{\mathbf{c}}(x)$ and $\rho_{\mathbf{C}}(x)$ it is convenient to introduce Green's functions (resolvents): $G(z)=(1 / N) \operatorname{Tr}(z-\mathbf{C})^{-1}$, where $z$ is a complex variable, and correspondingly $g(z)=$ $(1 / N)\left\langle\operatorname{Tr}(z-\mathbf{c})^{-1}\right\rangle$, where the average is over $\mathbf{X}$ from a Wishart ensemble with the correlations given by (11). It is also convenient to introduce generating functions for the spectral moments [5]:

$$
M(z)=\sum_{k=1}^{\infty} \frac{M_{\mathbf{C} k}}{z^{k}}, \quad m(z)=\sum_{k=1}^{\infty} \frac{m_{\mathbf{c} k}}{z^{k}},
$$

where $M_{\mathbf{C} k}=\int \rho_{\mathbf{C}}(\lambda) \lambda^{k} d \lambda$ and analogously for $m_{\mathbf{c} k}$. The generating functions are directly related to the resolvents: $M(z)=z G(z)-1$ and $m(z)=z g(z)-1$.

One can show [5] that if $N, T \rightarrow \infty$ with fixed $r=$ $N / T$, the function $m(z)$ can be calculated from $M(Z)$ as follows:

$$
m(z)=M(Z)
$$

where:

$$
z=Z(1+r M(Z))
$$

Assume that we know the correlation matrix $\mathbf{C}$. We can calculate eigenvalues of $\mathbf{C}, \lambda_{k}$ and their multiplicities $n_{k}$ (degeneracies), and determine $M(Z)$ :

$$
M(Z)=\sum_{k=1}^{K} \frac{p_{k} \lambda_{k}}{Z-\lambda_{k}},
$$

where $p_{k}=n_{k} / N$ is the fraction of all eigenvalues taking the value $\lambda_{k}$. For the later convenience we also assume that the eigenvalues are ordered $\lambda_{1}<\cdots<\lambda_{K}$.

For the given $M(Z)$ we can apply the equations (3) and (4) to determine $m(z)$ and $g(z)=(1+m(z)) / z$ and further, from $g(z)$ we can calculate the eigenvalue density $\rho_{\mathbf{c}}(x)$ by taking the imaginary part of $g(z)$ along the cuts on the real axis: $\rho_{\mathbf{c}}(x)=-(1 / \pi) \operatorname{Im} \mathrm{g}\left(\mathrm{x}+\mathrm{i} 0^{+}\right)$.

The equations (31) and (4) are equivalent to the Marčenko-Pastur equation [7]. Let us now discuss their physical content. 

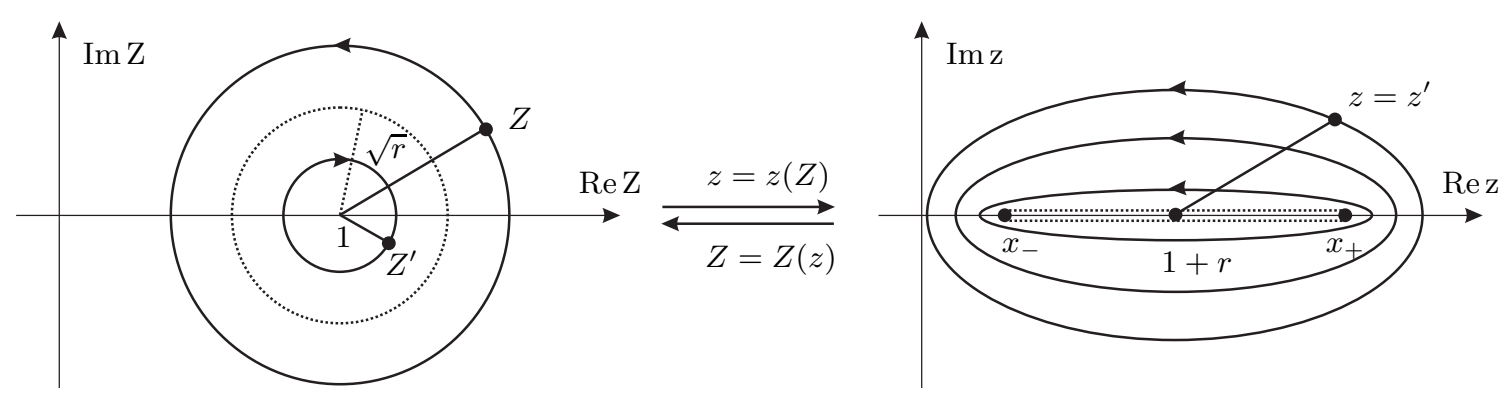

FIG. 1: Graphical representation of the map $z=z(Z)$. Each pair of points: $Z=1+R e^{i \phi}$ and $Z^{\prime}=1+(r / R) e^{-i \phi}$ is mapped onto the same point $z=z^{\prime}$. While $\phi$ increases, $Z$ and $Z^{\prime}$ move along the circles in the directions given by arrows. The critical horizon (dotted circle) has radius $\sqrt{r}$. The image of a circle $|Z-1|=R$ is an ellipse in $z$-plane, which for $R \rightarrow \sqrt{r}$ degenerates to an interval (dotted line).
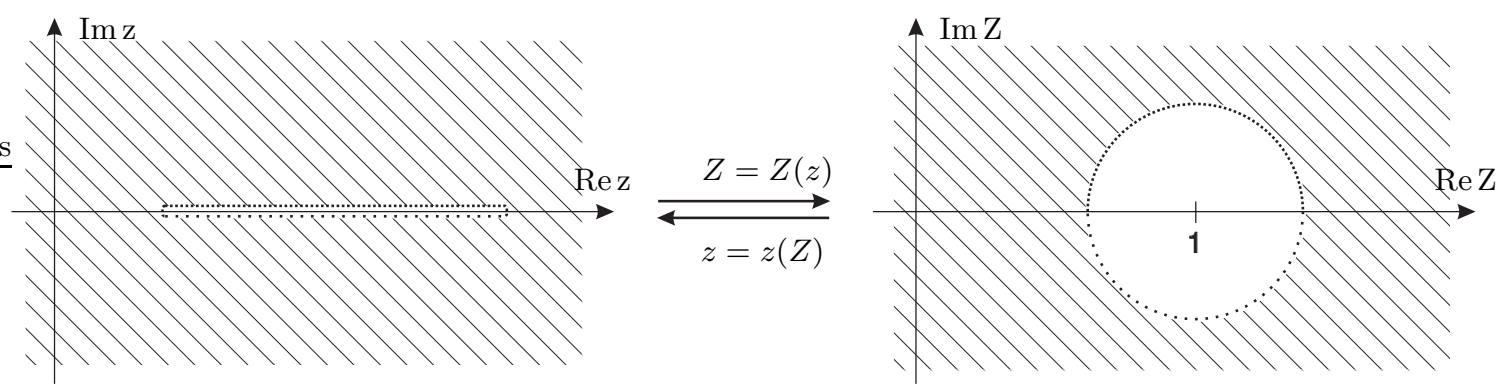

FIG. 2: The inverse function $Z=Z(z)$ : the $z$-plane without the real interval $\left[x_{-}, x_{+}\right]$is mapped onto the outside of the critical circle in the $Z$-plane. The interval is mapped into the upper (or lower) part of the critical semicircle.

The equation (31) tells us that the function $m(z)$ assumes for $r=0$ the same form as the function $M(Z)$ since in this case $z=Z$ as follows from the Eq. (4).

In this case the spectrum of the experimental matrix $\mathbf{c}$ is identical as of the matrix $\mathbf{C}$, as expected for $T \rightarrow \infty$. However for $r>0, z=z(Z)$ is a nontrivial function and the relation of the generating function $m(z)$ to the original one $M(Z)$ is not simple. The function $M(Z)$ has poles on the real axis at $\lambda_{k}$ 's. We may ask whether one can see the traces of the poles also in the function $m(z)$.

In order to answer this question first consider the simplest example $\lambda_{1}=\lambda_{2}=\ldots=\lambda_{N}=1$. The function $M(Z)=1 /(Z-1)$ has only one pole and the conformal map (4) takes the form:

$$
z=Z\left(1+\frac{r}{Z-1}\right)
$$

Consider a point on the $Z$-plane in the distance $R$ from the pole: $Z=1+R e^{i \phi}$. The function (6) assumes the value $z=1+r+R e^{i \phi}+(r / R) e^{-i \phi}$ the same as for a point $Z^{\prime}=1+(r / R) e^{-i \phi}$. As a consequence each point $Z$ outside the circle $|Z-1|=\sqrt{r}$ has a partner $Z^{\prime}$ inside this circle such that $z(Z)=z\left(Z^{\prime}\right)$ as depicted in Fig. 10 On the limiting circle there are pairs of points: $Z=1+\sqrt{r} e^{i \phi}$ and $Z^{\prime}=\bar{Z}=1+\sqrt{r} e^{-i \phi}$ for which $z$ assumes the same values. We see that the upper part of the semicircle is mapped onto an interval $\left[x_{-}, x_{+}\right], x_{ \pm}=(1 \pm \sqrt{r})^{2}$ on the real axis in the $z$-plane, and so is the lower semicircle.
Inverting the function $z=z(Z)$ one obtains a two-valued function $Z=Z(z)$ and thus one has to decide which Riemann sheet to choose. The physical Riemann sheet corresponds to the outside of the circle $|Z-1|=\sqrt{r}$ as follows from the expansion (2). For the inverse function $Z=Z(z)$ the image of the whole $z$-plane is given by the outside of the circle $|Z-1|=\sqrt{r}$ plus the upper semicircle which is an image of the cut (see Fig. 2). We thus see that the pole in the $Z$-plane is surrounded by a critical horizon behind which the argument of the inverse function $Z=Z(z)$ never enters. Only when the horizon radius $\sqrt{r}$ shrinks to zero the variable $Z$ can approach the "naked" pole.

As mentioned before, both semicircles of the critical horizon $|Z-1|=\sqrt{r}$ are mapped by the transformation (44) onto the same interval $\left[x_{-}, x_{+}\right]$on the real axis which makes the inverse function $Z=Z(z)$ two-valued. To make out of it a single-valued one one has to restrict the image to either the upper or lower semicircle. This can be done by approaching the cut from above: $z=x+i 0^{+}$ to obtain the upper semicircle or from below $z=x+i 0^{-}$ for the lower semicircle (Fig. 2). The imaginary part of $g(z)=(1+M(Z(z))) / z$ gives, for $z=x+i 0^{+}$, the eigenvalue density $\rho_{\mathbf{c}}(x)$.

We see that the shape of the eigenvalue density $\rho_{\mathbf{c}}(x)$ is encoded in the behavior of the conformal map (4) near the critical horizon. This is true in the general case (5). The poles of $M(Z)$ are distributed on the positive real 
semi-axis at $\lambda_{k}$ 's. For $r>0$ they are shielded behind a critical horizon (see Fig. 31). In general there are $K+1$ Riemann sheets. We are interested in the one for which $1 / z \rightarrow 0$ when $1 / Z \rightarrow 0$ in Eq. (2). Thus the physical region lies outside the critical horizon. It is symmetric about the real axis. The upper part $(\operatorname{Im} Z>0)$ is mapped by Eq. (4) onto intervals on the real axis in the $z$-plane and so is the lower one. When $r$ is positive but very small each pole has its own horizon, but when $r$ is increased the individual horizons grow and merge so that the number of connected components of the total horizon decreases with $r$ (see Fig. 3). For sufficiently large $r$ a single critical horizon surrounds all poles. The corresponding image of the horizon on the $z$-plane has a single cut on the real axis. For the limiting case $r=1$, the cut touches the origin at $z=0$.

The discussion presented above can be turned into a practical method of determining the shape of the eigenvalue distribution $\rho_{\mathbf{c}}(x)$. We have to find the inverse function $Z=Z(z)$ to $z=z(Z)$ (4), insert the solution to $M(Z)$ and determine the behavior of the resulting function along the cuts on the real axis. The equation (44) can be analytically solved for $Z$ only in few cases. In the general case one has to use a numerical method. Fortunately, one can bypass the problem of the explicit function's inversion by using a method described below.

In the first step we determine the critical horizon. Then moving gradually along its upper part $(\operatorname{Im} Z \geq 0)$ we calculate $z=z(Z)$ (4) which is in this case real $(z=$ $\left.x+i 0^{+}\right)$and simultaneously $\hat{\rho}_{\mathbf{c}}(Z)=-(1 / \pi) \operatorname{Im}(\mathrm{M}(\mathrm{Z})+$ $1) / z(Z) \equiv-(1 / \pi) \operatorname{Im}(m(z)+1) / z$ which gives the eigenvalue density $\rho_{\mathbf{c}}(x)=\hat{\rho}_{\mathbf{c}}(Z)$. Briefly speaking, the method is to use the auxiliary variable $Z$ on the upper part of the horizon to parametrize both the eigenvalue $x=z(Z)$ and $\rho_{\mathbf{c}}(Z)$, and to treat the pairs $\left(x(Z), \hat{\rho}_{\mathbf{c}}(Z)\right)$ as $\rho_{\mathbf{c}}(x)$. If the horizon has many disconnected parts, also the cut and the support of the eigenvalue density function $\rho_{\mathbf{c}}(x)$ consists of many disconnected intervals.

Let us describe how to determine the critical horizon. If $Z=X+i Y$ is a point on the horizon, the imaginary part of the map $z=z(Z)$ (4) vanishes and we have:

$$
\sum_{k=1}^{K} \frac{p_{k} \lambda_{k}^{2}}{\left(X-\lambda_{k}\right)^{2}+Y^{2}}=\frac{1}{r} .
$$

This equation has to be solved for $Y=Y(X)$ for given $X$. If the real solution exists it has two symmetric roots $\pm Y$. We are interested in the non-negative one $Y \geq 0$ which corresponds to $Z$ on the upper part of the critical horizon. Now we can calculate $x=z(Z)$ and $\rho_{\mathbf{c}}(Z)$ :

$$
x(Z)=X+r \sum_{k=1}^{K} p_{k} \lambda_{k}+r \sum_{k=1}^{K} \frac{p_{k} \lambda_{k}^{2}\left(X-\lambda_{k}\right)}{\left(X-\lambda_{k}\right)^{2}+Y^{2}},
$$

and

$$
\rho_{\mathbf{c}}(Z)=-\frac{Y}{\pi x} \sum_{k=1}^{K} \frac{p_{k} \lambda_{k}}{\left(X-\lambda_{k}\right)^{2}+Y^{2}}
$$

which, after ignoring $Z$ being a parameter, gives the pair $\left(x, \rho_{\mathbf{c}}(x)\right)$. The two equations above are explicit, so one can directly compute $x$ and $\rho_{\mathbf{c}}$ for any given $Z$ if one knows the dependence $Y(X)$. This dependence can be obtained by solving the Eq. (7) for $Y(X)$.

The solution of Eq. (7) depends on $r$. The function on the left-hand side of (7) has a shape of a 'hilly' landscape when plotted on $Z$-plane, with $K$ peaks of infinite height located at the poles $\left(\lambda_{k}, 0\right)$. The circumference of each peak shrinks to zero for increasing altitude. The solution of the equation lies on curves obtained as a cross-section of the hilly landscape at height $1 / r$. One can think of a coast line around islands surrounded by a sea with the water level $1 / r$. For $r \rightarrow 0^{+}$, the level goes to infinity, and thus the cross-section contains only the points at which the peaks are located: $\left(\lambda_{k}, 0\right)$. For $r>0$, the level $1 / r$ is finite, so the cross-section contains closed lines surrounding the poles and forming the critical horizon. When the water level drops the islands merge and the coast line grows (Fig. B).

One can find the points where the horizon crosses the real axis by setting $Y=0$ in Eq. (77). Among those points, the leftmost $X_{-}$and the rightmost $X_{+}$set the limits on the minimal and maximal value of $X$ where one has to look for the horizon solution. The two points are mapped by Eq. (44) onto $x_{ \pm}$being the lower and upper edge of the eigenvalue spectrum $\rho_{\mathbf{c}}(x)$. The values $X_{-}$ and $X_{+}$can be easily found by a root finder algorithm applied to the Eq. (17) with $Y=0$. Having determined $X_{-}$and $X_{+}$one can change $X$ in small steps from $X_{-}$to $X_{+}$and for each $X$ find the positive solution $Y$ of Eq. (7) to eventually determine $Z=X+i Y(X)$ on the horizon. In that way the problem is solved.

Let us now discuss examples. First we consider a correlation matrix $\mathbf{C}$ having three eigenvalues $\{1,2,6\}$ with equal weights $p_{k}=1 / 3$. The critical horizon for different $r$ as well as corresponding eigenvalue spectra $\rho_{\mathbf{c}}(x)$ are shown in Fig. 3 The evolution of the critical horizon with $1 / r$ can be treated as a level map for the landscape (7). In the inset of Fig. 3 we compare the spectrum for $r=1 / 3$ with a spectrum obtained by diagonalization of $n=3 \cdot 10^{5}$ matrices of size $N=48$ and $T=144$ chosen randomly from the corresponding Wishart ensemble. The agreement is perfect, up to a finite-size corrections near the edges of the cut.

The second example is related to some practical problem. The portfolio selection is a central problem of quantitative finance. The importance of the random matrix theory for this problem has been recently discovered [3]. It has been realized that the lower part of the spectrum $\rho_{\mathbf{c}}$ has a universal shape stemming from statistical fluctuations. The method which we described above gives a refined tool allowing one to observe a fine structure of the spectrum of the empirical matrix also in its lower part. As an example we consider a correlation matrix obtained for returns of 18 stocks on the Polish Stock Market. The eigenvalues read: $0.36,0.38,0.47,0.48,0.56,0.59,0.64$, $0.66,0.68,0.71,0.78,0.83,0.89,0.94,0.95,1.08,1.16$, 

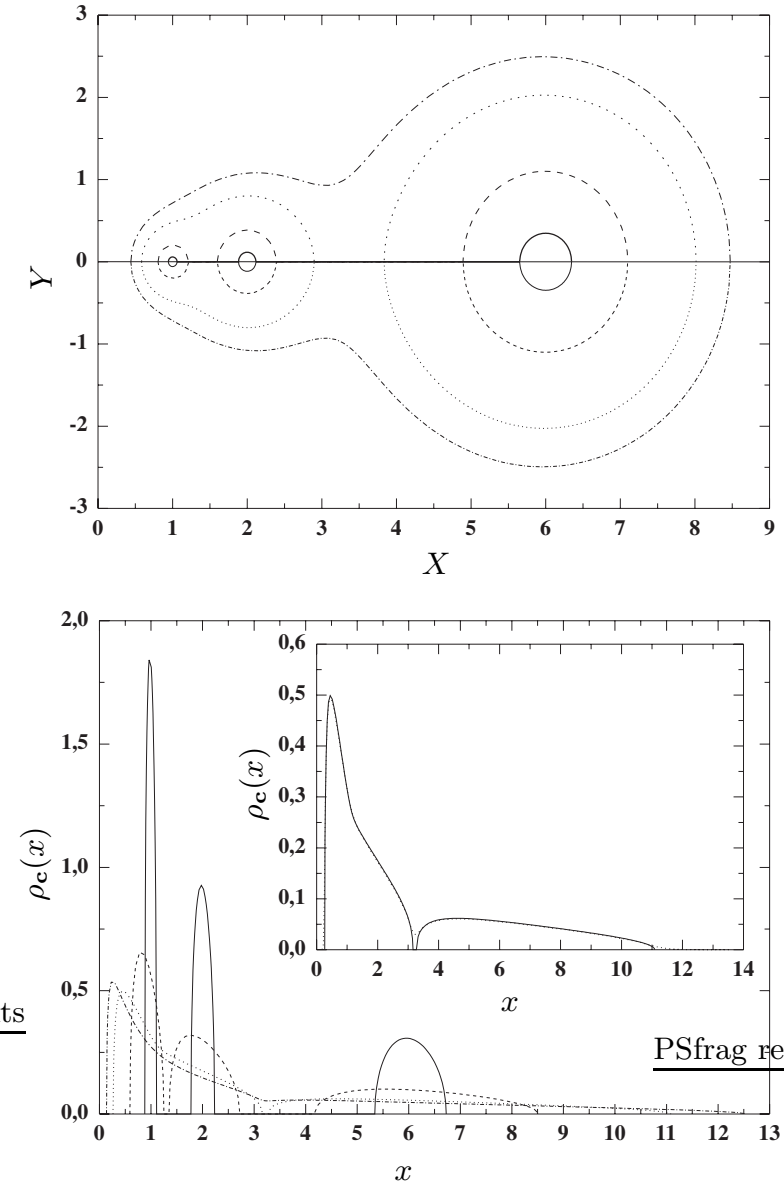

FIG. 3: Top: Critical horizon for $\left\{\lambda_{i}\right\}=\{1,2,6\}$ and for $r=$ $1 / 100$ (solid), $r=1 / 10$ (dashed), $r=1 / 3$ (dotted) and $r=$ $1 / 2$ (dash-dot). Bottom: The corresponding spectra $\rho_{\mathbf{c}}(x)$. Inset: the spectrum for $r=1 / 3$, calculated (solid line) and found experimentally (dotted) for sample of $3 \times 10^{5}$ matrices of size $N=48$.

5.84, they are normalized to $\sum_{k} \lambda_{k}=N$. In Fig. 4 we show the expected eigenvalue spectrum of $\mathbf{c}$ for $N=18$ and for $T=54(r=0.333)$ and $T=255(r=0.0706)$. For small $r$ a fine structure emerges in the spectrum. In figure the spectrum is also compared to the one obtained by diagonalization of $n=3 \times 10^{5}$ correlated Wishart ma- trices. The agreement is very good. This means that already for $N$ of order 20 the large $N$ limit, in which the analytical equations have been derived, applies. Deviations are observed only at the edges of the individual parts of the spectrum.

Statistical properties of random matrices and complex analysis are very intertwined. We used here this interrelation, or more precisely a conformal map representation of the Marčenko-Pastur equations, to derive a general, practical method for the determination of the eigenvalue distribution for the standard estimator of the covariance matrix. This method can also be applied to the corresponding equations [10] for the case of a correlated sample where the delta function $\delta_{\alpha \beta}$ in Eq. (10) is substituted

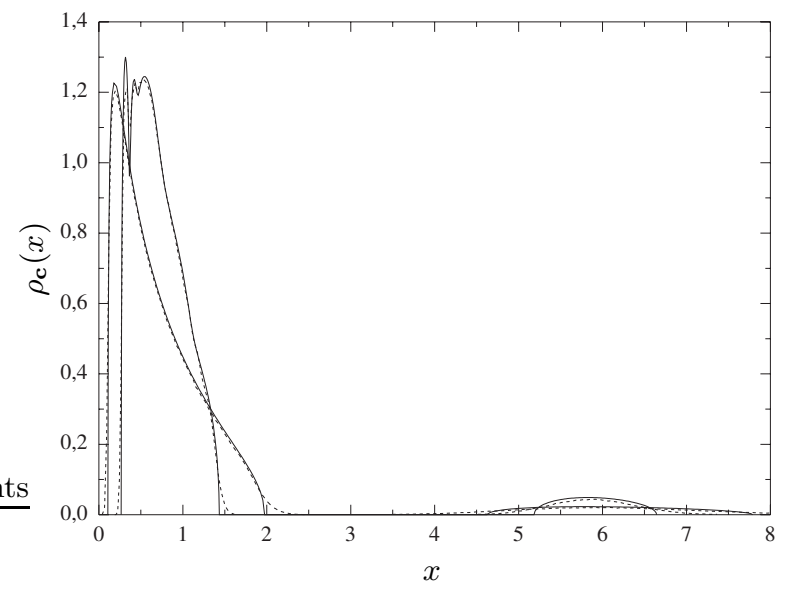

FIG. 4: Solid line: $\rho_{\mathbf{c}}(x)$ for $N=18$ eigenvalues of $\mathbf{C}$ taken from Polish Stock Market, for $T=54 \leftrightarrow r=0.333$ and $T=255 \leftrightarrow r=0.0706$, calculated using the method described in this paper. Dashed line: $\rho_{\mathbf{c}}(x)$ obtained by diagonalization of $3 \cdot 10^{5}$ Wishart matrices generated by a Monte-Carlo procedure.

by a correlation matrix $A_{\alpha \beta} \neq \delta_{\alpha \beta}$.

This work was partially supported by the Polish State Committee for Scientific Research (KBN) grant 2P03B08225 (2003-2006) and Marie Curie Host Fellowship HPMD-CT-2001-00108 and by EU IST Center of Excellence "COPIRA".
[1] R. Müller, IEEE Transactions on Information Theory, 48, 2495 (2002).

[2] S.E. Skipetrov, Phys. Rev. E67, 036621 (2003).

[3] J.P. Bouchaud, P. Cizeau, L. Laloux and M. Potters, Phys. Rev. Lett. 83, 1467 (1999).

[4] P.P. Mitra and A.M. Sengupta, Phys. Rev. E60, 3389 (1999).

[5] Z. Burda, A. Görlich, A. Jarosz, J. Jurkiewicz, Physica A343, 295 (2004).

[6] J. Wishart, Biometrika A20, 32 (1928).

[7] V.A. Marčenko and L.A. Pastur, Math. USSR-Sb. 1, 457
(1967)

[8] Z.D. Bai and J.W. Silverstein, J. Multivariate Anal. 54, 175 (1995).

[9] S.I. Choi and J.W. Silverstein, J. Multivariate Anal. 54, 295 (1995).

[10] Z. Burda, J. Jurkiewicz and B. Waclaw, Phys. Rev. E71, 026111 (2005).

[11] J. Feinberg, A. Zee, Jour. Stat. Phys. 87, 473 (1997).

[12] For simplicity we will assume that $\forall i:\left\langle x_{i}\right\rangle=0$.

[13] One can also consider complex matrices. The large $N$ limit is in this case identical as for real matrices. 